\title{
Optimizing Gaze Typing with Pupil Size for People with Severe Motor Disabilities
}

\author{
Xiangsheng Wang, \\ Zhangqiu hospital of traditional Chinese medicine CT chamber, \\ Shandong, China. \\ E-mail:sdzqzyy@sina.com
}

\begin{abstract}
Thanks to recent technological advances in the field of eye tracking, eye typing provides means of communication for people with severe disabilities. Typing with gaze using dwell time has been made possible by the development of eye tracking technologies. Recent research indicates that pupil size is viewed as a subtle cue of people is making a decision. Therefore, it may help to infer users' willing of typing. The present study describes the design process for improving eye typing by adding pupil size index into dwell time triggering. Experimental evaluations showed that the approach was effective; design considerations for such optimization of the gaze typing interfaces are discussed.
\end{abstract} size

Keywords- eye-typing; severe disabilities; dwell time; pupil

\section{I . INTRODUCTION}

As early as 1982, Bolt introduced using eye-gaze as input to facilitate HCI [1]; numerous studies have been conducted on utilizing eye movements to trigger operations. The theme of gaze input is being more and more considered nowadays, especially in the context of user with motor disabilities. People affected by severe motor disabilities need ways to interact with apparatus, which can provide them an essential communication means. Eye typing is a basic scenario of eye gaze input [2]. Due to advances in eye tracking hardware and software, eye typing systems are becoming increasingly used. Moreover, it has been proved that gaze typing could be implemented in personal computer setting. Abe et al. [3] developed a gaze typing system which utilizes a personal computer and a home video camera to detect eye-gaze under natural light. In their experiment, subjects can input text at an average rate of 16.2 characters per min by properly use the eye movements history.

However, Typing with the eyes has tough problems than typing with fingers, one is eye typing does not allow parallel processing, the distances between the on-screen keyboard are crucial [4]; the other is eyes can not give a clear command, because eye movements are both conscious and unconscious, consequently, computers have to deduce users' intention. In order to insure an action, nowadays, in eye typing scenarios, actions initiate almost by two types of solutions. One is adding operations, such as providing the user with a manual control key for activation or blinking on the fixation. The ideas of using manual control key are not so convenient; eye blinks are proved to be impractical, for it is hard for users to exercise precise enough to control over the blink reflex [5]. The other most commonly used approach is to prolong the dwell time for triggering, the user has to fixate an item for a predefined period of time in order to activate it. Input based on dwell time has to face the problem of finding the optimum threshold for reasons: long threshold of duration fatigues the user, while short threshold are easy to result in mistake, that is, information examine behavior is easy to interpret as input command (namely the Midas touch [6]) in such situation.

In order to overcome the shortcoming of dwell input, supplementary solutions have been proposed. One is to use the history of gaze, in the method of Abe, they divided the gaze state into two states: the initial state and the continuous state. If the users gaze at an indicator more than a defined period of time, then define this state as the initial state. Continuous state is defined as the state follow after the initial state. Majority numbers of gazed indicator in each state are extracted as the candidate input. If two indicators are equal, the candidate input is selected. Otherwise initiate another turn. Another is to combine Brain-Computer Interface (BCI) with eyes for more precise triggering. For example, Zander et al. combine the electroencephalogram (EEG) signal and eye-tracker dwell time for a characters search-and-select task. The result showed that pooled BCI accuracy was 83.3\% [7]. However, the implementation and preparation for such method is at the great expense.

\section{II . OPTIMIZING GAZE TYPING WITH PUPIL SIZE TO SUPPLEMENT DWELL TIME}

Spontaneous fluctuations in pupil size have been used as dependent variables in psychological investigations. Pupil dilation has been implicated as a marker for decision processes. Pupil dilation has been found when participants making decision and a short of time after decision [8]. The pupil dilation appeared to reflect the post-decisional consolidation of the selected outcome. Moreover, researchers have utilized pupil response to do some prediction works. For example, Carter et al. could predict with 62 to 100 per cent accuracy (depending on the volunteer) which of the five intervals people would choose in experimental task, only based on when their pupil dilated [9]. Norephinephrine may play a specific role in consolidating behavioral decision [10], and there is a tight link between pupil dilation and norepinephrine levels during constant illumination, it can explain why pupil size related to decision processes. 
In this paper, we discuss the prospect of utilizing pupil size index to help infer the input initiate intention of users, so as to better assist gaze typing for people with severe motor disabilities.

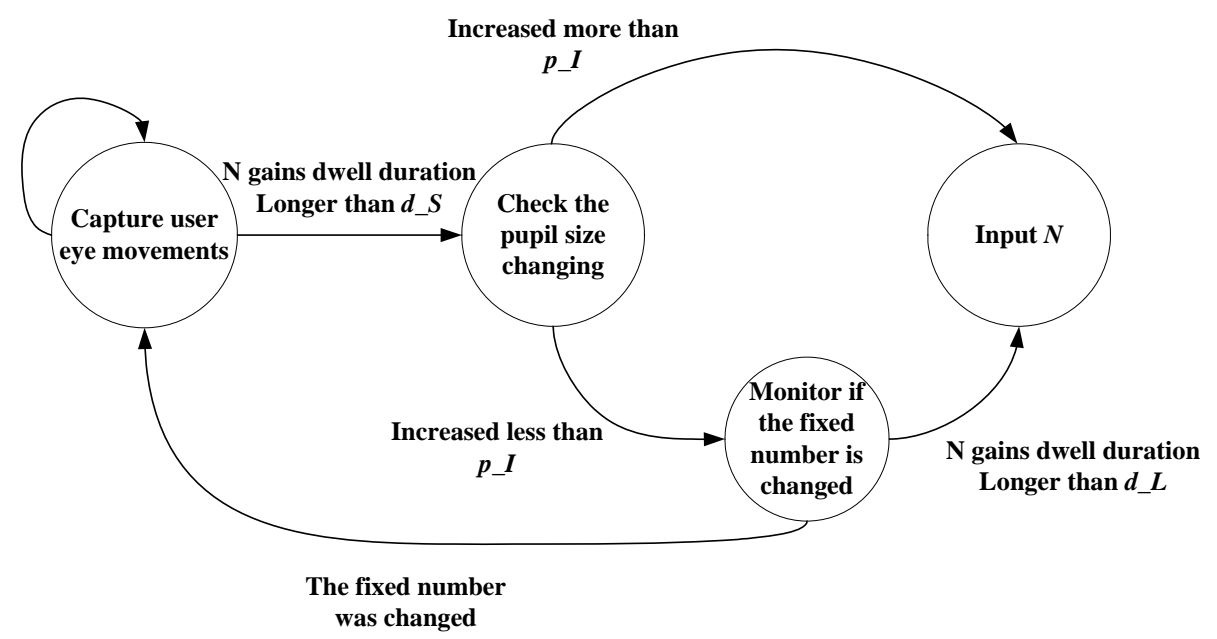

Fig. 1 Illustration of adding pupil size to dwell time number input

As show in Fig. 1, for the first phase normal pupil size during individual information examining is detected in a period of time. Next, two dwell threshold are defined, a shorter one $d \_S$ and a long one $d_{-} L$. When a number $N$ gains dwell duration longer than $d_{-} S$, $\mathrm{N}$ highlighted, then check whether the pupil size exhibit an increase by $p_{-} I$ during the $50 \mathrm{~ms}$ latter, if it is true then input the number fixated, otherwise waiting the dwell duration reach $d_{-} L$ and the $\mathrm{N}$ highlighted, and trigger input after 50ms. If the dwell duration do not reach $d \_L$ and the object user fixate is changed, initiate another turn.

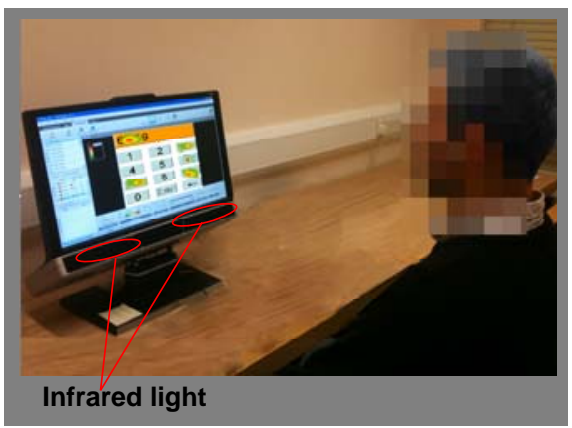

Fig. 2 Experimental Apparatus

\section{EXPERIMENTAL EVALUATION}

The eye-tracker employed in this research was Tobii t120 made in Sweden. Tobii t120 is non-intrusive, looked like a regular computer screen, it tolerance of large head movements allows subjects to move freely and naturally (as shown in Fig. 2). The Tobii studio serve as the software present the numbers automatically and recorded the data during participants typing numbers.

Suppose $x \in[a, b]$ is the coordinate value of $x$ which obtained from eye-tracker, we can map it into [c, $d]$ according follow formulation:

$$
x^{\prime}=c+\frac{(x-a)(d-c)}{(b-a)}
$$

The follow formulations can map eye-tracking coordinate $(x, y)$ into graph coordinate $\left(x^{\prime}, y^{\prime}\right)$, parameters in which are shown in Fig.3.

$$
\begin{aligned}
& x^{\prime}=\text { left }+\frac{x(\text { right }- \text { left })}{512} \\
& y^{\prime}=\text { bottom }+\frac{(512-y)(\text { top }- \text { bottom })}{512}
\end{aligned}
$$

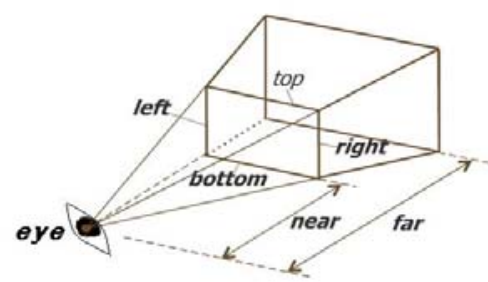

Fig.3 The mapping of eye tracking system coordination into 3D scene

Suppose the distance between two eyes is $b$, the coordinate of fixation near clip plane for the left eye is $\left(x_{l}, y_{l}\right)$, right eye is $\left(x_{r}, y_{r}\right)$, the distance between eyes 
and near clip plane is $f$, the center of two eyes' coordinate $\left(x_{h}, y_{h}, z_{h}\right)$ can be calculated as follows:

$$
\begin{aligned}
& x_{g}=(1-s) x_{h}+s\left(\left(x_{l}+x_{r}\right) / 2\right) \\
& y_{g}=(1-s) y_{h}+s\left(\left(y_{l}+y_{r}\right) / 2\right) \\
& z_{g}=(1-s) z_{h}+s f
\end{aligned}
$$

where,

$$
s=\frac{b}{x_{l}-x_{r}+b}
$$

Reference to previous works [11], we setted the distance between numbers with $1.1^{\circ}$, the size of numbers with $1.6^{\circ}$. Fig. 4 shows the gaze input keyboard of our study, there are totally 12 keys, in which character " 5 " was highlighted. At first we recruited 10 participants to test the pupil size changing and dwell pattern of this keyboard, thus to assess p_I. They were instructed to look at pre-defined characters and chose by mouse click.

Reference to [11], we defined two $d_{-} S$ and $d_{-} L$ candidaters, respectively. Therefore, There were 8 settings of our experment, $d \_S(300 \mathrm{~ms}, 400 \mathrm{~ms}) \times$ $d \_L(600 \mathrm{~ms}, 700 \mathrm{~ms}) \times p \_I(0.021 \mathrm{~mm}, 0.032 \mathrm{~mm})$. Three participants sit in a wheel chair, the distance between chair and screen was about $60 \mathrm{~cm}$. Participants needed to input 6 phone numbers, include 3 mobile phone numbers (13510276784, 13098644327, 18901097568) and 3 fixed telephone numbers
(67346210, 56084532, 45027688), and they need to end with "Enter", also can change inputted number with “ऍ”.

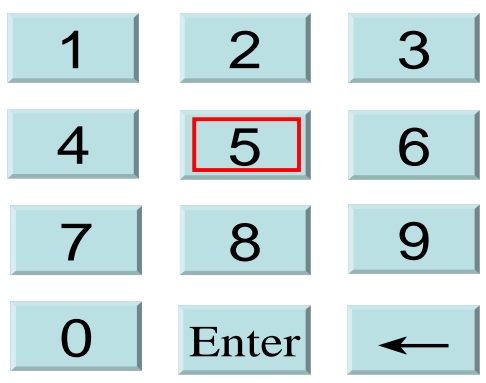

Fig. 4 The gaze input keyboard

Table 1 shows the input time under difference conditions. When $d \_S=300 \mathrm{~ms}, d_{-} L=600 \mathrm{~ms}, p_{-} I=$ $0.021 \mathrm{~mm}$, the input rate reach the highest level, no matter the objects is mobile phone or fixed phone numbers. Since there are at least 12 keys for input a mobile phone number and 9 keys for a fixed phone number, the average rate for input a character is $638 \mathrm{~ms}$ for the mobile phone number, 618ms for the fixed phone number. As shown in table 2, in general, the error rate is negative correlate to the difference between $d_{-} S$ and $d_{-} L$. there are no significant differences between $L_{p_{-} I}$ and $S_{p_{-} I}$ with the error rate.

Table 1 Input time under difference conditions

\begin{tabular}{ccccccccc}
\cline { 2 - 8 } \begin{tabular}{c} 
Dwell time(ms) \\
\cline { 2 - 8 } Pupil size(mm)
\end{tabular} & \multicolumn{4}{c}{ Mobile phone number } & \multicolumn{5}{c}{ Fixed phone number } \\
\cline { 2 - 9 } & $400-600$ & $400-700$ & $300-600$ & $300-700$ & $400-600$ & $400-700$ & $300-600$ & $300-700$ \\
\hline 0.021 & $7.93 \mathrm{~s}$ & $8.67 \mathrm{~s}$ & $7.65 \mathrm{~s}$ & $8.38 \mathrm{~s}$ & $6.21 \mathrm{~s}$ & $6.32 \mathrm{~s}$ & $5.56 \mathrm{~s}$ & $5.92 \mathrm{~s}$ \\
0.032 & $8.07 \mathrm{~s}$ & $8.56 \mathrm{~s}$ & $7.92 \mathrm{~s}$ & $8.71 \mathrm{~s}$ & $6.46 \mathrm{~s}$ & $6.81 \mathrm{~s}$ & $6.78 \mathrm{~s}$ & $6.31 \mathrm{~s}$ \\
\hline
\end{tabular}

Table 2 Error rate under difference conditions

\begin{tabular}{ccccccccc}
\cline { 2 - 8 } \begin{tabular}{c} 
Dwell time(ms) \\
\cline { 2 - 9 } Pupil size(mm)
\end{tabular} & $400-600$ & $400-700$ & $300-600$ & $300-700$ & $400-600$ & $400-700$ & $300-600$ & $300-700$ \\
\hline 0.021 & $12.1 \%$ & $5.6 \%$ & $6.2 \%$ & $3.8 \%$ & $9.8 \%$ & $4.3 \%$ & $5.5 \%$ & $2.9 \%$ \\
0.032 & $10 \%$ & $5.3 \%$ & $5.9 \%$ & $4.0 \%$ & $8.6 \%$ & $3.9 \%$ & $5.4 \%$ & $3.3 \%$ \\
\hline
\end{tabular}

\section{CONCLUSION}

The present study explored the effectiveness of combining the pupil size into the classical dwell threshold triggering method. Cognitive scientist found that when participants making decision and a short of time after decision their pupil dilated. By utilizing this phenomenon, we successfully improve the phone number input rate for people with severe disabilities. Our method have limitations, the pupil size is very sensitive, it affected by many factors such as colors, size and backgrounds of the items, this method may fail to make sense in situations of object are complex. Thus our future works involved extracted such influential factors and set parameter for them to broad the usable scenarios of introduced method.

\section{REFERENCES}

[1] Bolt, R. A.: Eyes at the interface. in Proceedings of the 1982 conference on Human factors in computing systems, 1982, 360-362. 
[2] Päivi Majaranta and Kari-Jouko Räihä. 2002. Twenty years of eye typing: systems and design issues. In Proceedings of the 2002 symposium on Eye tracking research \& applications (ETRA '02). ACM, New York, NY, USA, 15-22.

[3] Abe, K., Ohi, S., and Ohyama, M., “An eye-gaze input system using information on eye movement history”, In Proceedings of the 4th International Conference on Universal Access in Human-computer Interaction: Ambient Interaction, pp. 721-729, 2007.

[4] Areej Al-Wabil, Arwa Al-Issa, Itisam Hazzaa, May Al-Humaimeedi, Lujain Al-Tamimi, Bushra Al-Kadhi. Optimizing Gaze Typing for People with Severe Motor Disabilities: The iWriter Arabic Interface. ASSETS'12, October 22-24, 2012, 261-262.

[5] Hutchinson, T. F.: Eye-Gaze Computer Interfaces: Computers That Sense Eye Positions on the Display. Computer, 26, 1993, 65-67.

[6] Jacob, R. J. K.: What you look at is what you get: eye movement-based interaction techniques. in Proceedings of the SIGCHI conference on Human factors in computing systems: Empowering people (CHI '90), 1990, 11-18.

[7] Vilimek, R., Zander. T. O.: BC(eye): combining eyegaze input with brain-computer interaction. in Proceedings of the 5th International on Conference Universal Access in Human-Computer Interaction. Part II: Intelligent and Ubiquitous Interaction Environments, 5615, 2009, 593-602.

[8] Simpson, H. M., Hale, S. M.: Pupillary changes during a decision making task. Perceptual and Motor Skills, 29(2), 1969, 495-498.

[9] Einhauser, W., Koch, C., Carter, O.: Pupil dilation betrays the timing of decisions. Frontiers in Human Neuroscience, 4:18, 2010.

[10] Aston-Jones, G., Cohen, J.: An integrative theory of locus coeruleusnorepinephrine function: adaptive gain and optimal performance. Annual Review of Neuroscience, 28, 2005, 403-450.

[11] Chengzhi Feng. Eye-movement Based HumanComputer Interaction. Suzhou University press. 2010. 45-58.In Chinese 